\title{
Distributed Software Maintenance Using an Autonomic System Management Approach based on the Viable System Model
}

\author{
Emil A. Stoyanov \\ University of Stuttgart
}

\author{
Emil.Stoyanov@ \\ informatik-uni- \\ stuttgart.de
}

\author{
Asa MacWilliams \\ Siemens AG, \\ Corporate Technology
}

\author{
Asa.MacWilliams@ \\ siemens.com
}

\author{
Markus A. Wischy \\ Siemens AG, \\ Corporate Technology \\ Markus.Wischy@ \\ siemens.com
}

\author{
Dieter Roller \\ University of Stuttgart
}

\begin{abstract}
Most current software management solutions are missing a systematic and holistic approach for global system management, and apply only to specific system components. This limits the system's extensibility, and does not meet interoperability requirements from the growing heterogeneity of the operating environment. This paper discusses an alternative approach to common management of evolving distributed software, inspired by the research in the field of open systems and cybernetic models. The approach involves adapting Stanford Beer's Viable System Model (VSM) to the concrete needs of distributed software, supporting evolutionary integration of new functionality, while preserving system stability. We introduce meta-data bindings to existing information models as a basis for management of hierarchical and recursive software elements, and an architecture for composition of interacting components with verification of their capabilities during deployment and runtime. The architecture is designed to satisfy criteria of the Viable System Model and to assure conditions necessary for autonomic behavior.
\end{abstract}

\section{Introduction}

Deployment of new functionality in a software system is known to cause system regression. Research on this topic is going on for more than a decade and it becomes clear that testing a complex system after new functionality has been introduced is a difficult, time consuming and expensive task. Deployment of new components in distributed environment makes the task even harder because any model-based or statereduction regression testing method requires a large amount of resources and time to complete the actual number of tests to confirm system stability. Additionally, the modern IT environment utilizes loosely-coupled components and remote service execution that need implicit methods for verification of system integrity. Although there are results in the field of service composition, the problems of evolution and the effect of evolved components on the overall system stability in heterogeneous environment remains untouched. Similar and additional complexitymanagement related issues lead to the development of the Autonomic Computing and Communication initiatives.

An important standpoint in our research is the similarity between the characteristics of modern software systems and the open systems as seen by the General System Theory and Cybernetics - well established and proved by the time concepts related to understanding and control of biological and social systems $[9,14]$. Additionally, the extensive research on software evolution done by M. Lehman [10] confirms that software systems and their life-cycle are open, multi-loop, complex control systems and their management requires understanding of systems organization.

In order to operate on a system level we turned to the principles and methods for building viable systems for enterprises, extensively discussed in the research of Stanford Beer [12, 13], a recognized cybernetics researcher and contributor. Main properties of his Viable Systems Model (VSM) were already illustrated in relation to software development $[2,4,7]$. Our research is focused on handling problems related to system update. Upgrading a working system that consists of multiple distributed components is a risky and error-prone process. Service unavailability caused by conflicts between new versions of components and old ones is a common situation. This problem raises challenges related to management of the system as a whole and is directly related to the focus of Autonomic Computing. So far no concrete methodology and examples have been proposed to support holistic management and smooth system update of highlydistributed systems and we think VSM-based component management is a promising solution for this aim. 


\section{VSM Adaptation to Software Systems}

The Viable System Model (VSM) aims to reflect the organization of complex self-adaptive systems. In our understanding, its elements are just another manifestation of the main concept of the managed autonomic element[1]. First we will focus on the building blocks of a VSM system and after that on the requirements that the model defines as a criteria for viable system. We will describe on the first place the general approach of VSM and then its software implication.

\subsection{Viable System Components}

VSM defines several types of entities necessary to outline a viable system - environment, operation, management levels, and communication channels.

- Environment - the environment in which the system operates. It is the variety that is exposed to the system throughout its life. In general, the environment for a system is a set of foreign systems.

- Operation - unit responsible for the direct interaction with the environment

- Management - three types of management are distinguished:

- Controlling - manages the operation

- Planning - anticipates changes and estimates impact on the system, caused by change in the environment

- Executive - represents identity and defines the policies of the system.

- Communication Channels - an abstract term associated with the process of variety transmission and communication between components of a system. Communication channels can amplify (requires extra knowledge) variety or attenuate (filter) it.

- Variety of a system is defined by Cybernetics as the set of states a system may have. Every element in a system contains a subset of the system's variety. In the context of VSM and communication channels variety is considered a subset of states that an element transfers to another element. In this work we are considering the variety introduced by software components into the managed software system after their deployment or update.

\subsection{Viable System Requirements}

VSM sets requirements for system organization that guarantee viability - requisite variety (RV) of the channels and requisite knowledge (RK) of the management, RV of transducers, self-containment, and system responsiveness.

- $\quad \mathrm{RV}$ and RK of channels - assures that policies set by the executive management are properly transformed to actions plans of the operation.

- RV of transducers - transducers are entities that transform variety from one type into other. A transducer participating in channel communication is required to have at least the variety of the channel.

- System responsiveness and maintenance - the system should be able to handle the incoming variety from the environment without delay, otherwise the system may become unstable. This requirement is related to the need for enough capacity of the communication channels to be able to transmit enough variety in a corresponding period of time.

- Self-containment - the operational management is itself a viable system and follows the same organizational model as its parent system. This requirement assures common structure on all sublevels of system organization.

We have briefly presented the main characteristics of VSM. Applying such an abstract model in concrete and practical process such as run-time and deployment software management is not a trivial task. In the process of this adaptation we have taken the steps described below.

\subsection{Outlining Software System Entities}

We are interested in a class of software components that run and interact in distributed manner. Representatives of this class are component based and service oriented frameworks, such as Enterprise Java Beans (EJB), Components for provision of Web Services, OSGi[11] bundles, etc.. Each one of them is a sub-system of a parent container framework and each one of them is a subject of variation throughout system's life. The ongoing interaction between these types of components and their respective life-cycle management frameworks requires compatibility verification when it comes to update of a single component. Incompatibility between old versions of components and new ones may result in denial of service or can have deeper impact.

\subsection{VSM-Software Management Mapping}

In order to utilize VSM management with already selected and implemented management model there is a need of a proper model for mapping VSM entities to related entities of the selected model. It is important to 
note that the below proposed VSM utilization model is not and can not be used as substitution of any information model. It is a set of requirements and organization on a higher level that provides a management template that can be used by the management instrumentation of the selected information model. We have selected an approach to mapping of the model that is divided in three stages. First stage describes how entities located on same level of recursion relate to entities in the management model (Table. 1). The second stage maps the requirements of VSM that are valid in a single recursion level to entities described by the selected management model (Table 2). The third stage deals with the recursiveness that VSM systems exhibit and the possible relation types they can utilize (Table 3 ).

In order to keep examples close to reality we selected an already developed information model as a base for demonstration. There are several information models available $[5,6]$ that target IT management but as a promising and extensive one the Common Information Model (CIM), specified by DMTF has the potential to become standard for autonomic management systems [3]. Although CIM provides extensible schema for description of IT resources it does not tell more about concrete handling of management information. Table 1 illustrates sample relations between VSM entities, CIM selected elements and concrete IT elements that we are interested in.

\begin{tabular}{|l|l|l|}
\hline VSM Entity & \multicolumn{1}{|c|}{ Software Entity } & \multicolumn{1}{|c|}{ CIM Entities } \\
\hline Environment & $\begin{array}{l}\text { Network Resource, } \\
\text { Hardware resource, } \\
\text { Other Software } \\
\text { Systems }\end{array}$ & $\begin{array}{l}\text { SoftwareService, } \\
\text { PhysicalElement, } \\
\text { LogicalDevice }\end{array}$ \\
\hline Operation & $\begin{array}{l}\text { Execution Logic } \\
\text { Software Components }\end{array}$ & $\begin{array}{l}\text { SoftwareElement, } \\
\text { SoftwareFeature }\end{array}$ \\
\hline Management & $\begin{array}{l}\text { Component life-cycle } \\
\text { management, Network } \\
\text { Management, } \\
\text { Hardware management }\end{array}$ & $\begin{array}{l}\text { Application, } \\
\text { PhysicalElement, } \\
\text { Service Access Point }\end{array}$ \\
\hline $\begin{array}{l}\text { Communication } \\
\text { Channels }\end{array}$ & $\begin{array}{l}\text { Remote Service } \\
\text { Interfaces, } \\
\text { Network Management } \\
\text { Interfaces }\end{array}$ & $\begin{array}{l}\text { Dependency, } \\
\text { ServiceProvider, } \\
\text { Service Requester, } \\
\text { Endpoint Statistic } \\
\text { Service, SNMP } \\
\text { Service }\end{array}$ \\
\hline Variety & $\begin{array}{l}\text { Component Versions, } \\
\text { Interface signatures, } \\
\text { Configurations }\end{array}$ & $\begin{array}{l}\text { Capabilities, } \\
\text { Softwareldentity, } \\
\text { Setting }\end{array}$ \\
\hline
\end{tabular}

Table 1: Stage 1 - Mapping VSM to Software Entities
The second stage of model mapping involves definition of requirements for viable software system. It adds the needed semantics between actual software entities and VSM requirements that form the management scheme. The third necessary step to achieve full utilization of VSM requires is to examine how recursiveness appears in software components and how it is mapped to the recursive model offered by VSM. This would allow verification of variety transformation on a higher level as required by VSM (Table 2), e.g. dependencies between components of two heterogeneous frameworks that operate in the same logical system.

\begin{tabular}{|l|l|l|}
\hline VSM Requirement & Impact on Software & \multicolumn{1}{|c|}{ CIM Entities } \\
\hline $\begin{array}{l}\text { Channel Requisite } \\
\text { Variety }\end{array}$ & $\begin{array}{l}\text { Communicating } \\
\text { Components }\end{array}$ & $\begin{array}{l}\text { Component, } \\
\text { ServiceComponent, } \\
\text { Capabilities }\end{array}$ \\
\hline $\begin{array}{l}\text { Management } \\
\text { Requisite Knowledge }\end{array}$ & $\begin{array}{l}\text { Policy Specification, } \\
\text { Policy Execution }\end{array}$ & $\begin{array}{l}\text { PolicyCondition, } \\
\text { PolicyRule, } \\
\text { PolicyAction }\end{array}$ \\
\hline $\begin{array}{l}\text { System } \\
\text { Responsiveness }\end{array}$ & $\begin{array}{l}\text { Server Load } \\
\text { Optimization, } \\
\text { Configuration }\end{array}$ & $\begin{array}{l}\text { ComputerSystem } \\
\text { Resource,ElementCo } \\
\text { nfiguration, System } \\
\text { Statistics }\end{array}$ \\
\hline $\begin{array}{l}\text { Self-reference and } \\
\text { containment }\end{array}$ & $\begin{array}{l}\text { Server Clusters, } \\
\text { Server, Application } \\
\text { Container, } \\
\text { Application, Software } \\
\text { Component s }\end{array}$ & $\begin{array}{l}\text { Cluster, Computer } \\
\text { System, Application } \\
\text { System, Software } \\
\text { Element }\end{array}$ \\
\hline
\end{tabular}

Table 2: Mapping VSM Requirements to To Software Entities

Figure 1 illustrates a simple example for heterogeneous system consisting of three interacting components operating in two different containers. Bundle $A$ requires execution of remote method provided by the Web Service A. The two components are related logically but physically separated in different containers. In this setup we can notice several types of dependencies:

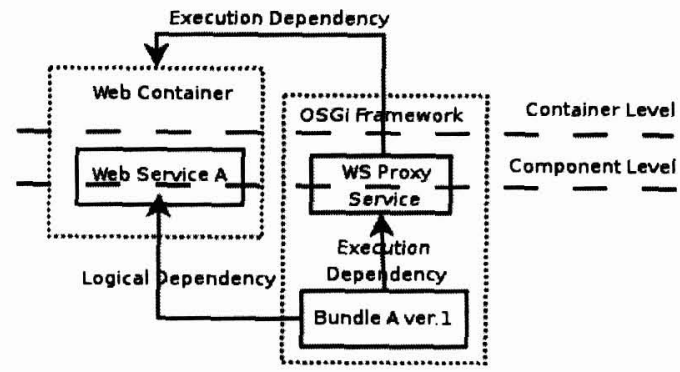

Figure 1. Example for dependencies in heterogeneous system 
a) Direct and indirect dependencies between components in same level of containment and same owner, e.g. respectively dependency of execution between OSGi bundle and local service provided by other bundle, logical dependency between two components.

b) Indirect dependencies between components on equal level of containment but different owner, e.g. logical dependency between bundle in OSGi Framework and Web Service located in remote web container.

c) Direct dependencies between elements and owner in different containment level, e.g. component relies on system services like component names lookup, event subscription, etc.

d) Indirect dependencies between component and foreign owner in different containment levels, e.g. fetching of WSDL descriptors from remote web container.

A clearer visual representation of these types of relation is shown on Fig. 2.

Similar relations between components in IT systems can be found on many levels of organization. CIM Specification defines composite systems and applications as separate classes. Applications are significantly narrowed abstraction of container aggregating features, software elements and deployment specifics while a CIM system as a class has a broader meaning of aggregation of components that operate to support both hardware peripherals, devices and and software applications. Table 3 shows the types of dependencies that a CIM-based management software can utilize with the help of VSM . The table includes only CIM basic groups of classes for simpler interpretation of the dependencies.

\begin{tabular}{|l|l|l|l|}
$\begin{array}{l}\text { Software } \\
\text { Entities }\end{array}$ & $\begin{array}{l}\text { Software } \\
\text { Component, } \\
\text { Software } \\
\text { Service }\end{array}$ & $\begin{array}{l}\text { Software } \\
\text { Application, } \\
\text { Service } \\
\text { Composition }\end{array}$ & $\begin{array}{l}\text { Component } \\
\text { Framework, } \\
\text { Server, Cluster }\end{array}$ \\
\hline CIM Entities & $\begin{array}{l}\text { Software } \\
\text { Element }\end{array}$ & Application & System \\
\hline $\begin{array}{l}\text { Software } \\
\text { Element }\end{array}$ & A, B & C, D & C, D \\
\hline Application & - & A & C \\
\hline System & - & - & A \\
\hline
\end{tabular}

Table 3: Stage 3- Mapping of level dependencies in software systems

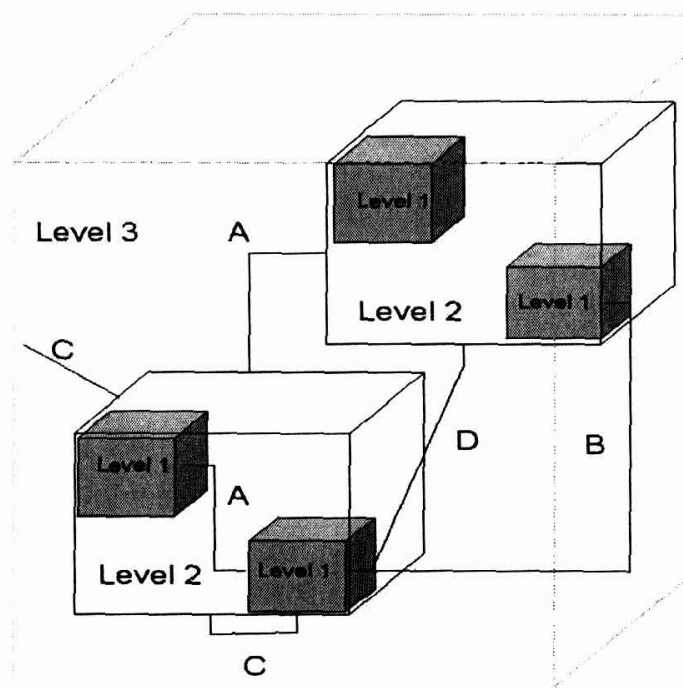

Figure 2. Type of relations between levels of containment in software components

With these three steps of model adaptation we have the opportunity to create management tools which use VSM as a reference model for management of complex hierarchical software structures.

\section{Enabling Viability}

In chapters 2.1 and 2.2 we outlined the requirements of VSM with the goal to make the system remain viable under the influence of incremental software updates which are the main source of introduced variety. While self-referencing support can be resolved using the proposed method in 2.4 , the main requirements for requisite variety of communication channels and channel transducers demand architectural approach reflecting the basic method of communication of VSM elements communication channels. Figures 3 and 4 illustrate our solution to channel-centric design of software component communication. A central role of managing variety exchange between components is given to a common channel framework that can be embedded into component frameworks by means of subscription for notification of system events, such as deployment and update of components or notification for registration of services. Meta-data related to the management and needed management information is stored separately in external knowledge base. This knowledge base is shared among software elements participating in the system process that the IT system supports. 


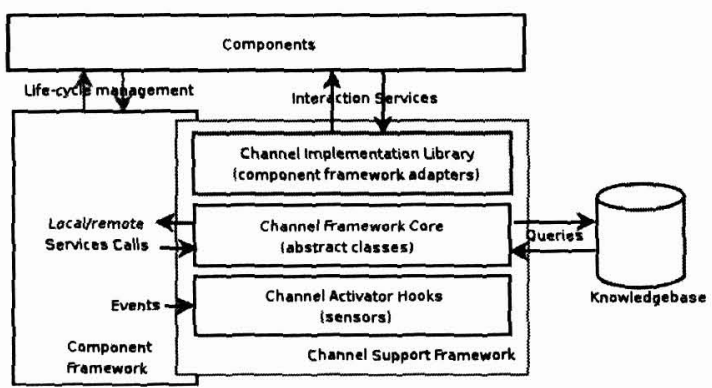

Figure. 3 Channel-centric communication management

It is important to note that the channel management module does not affect in any way the life-cycle management function of the framework. It depends on the component if connection with services and other components is established via the channel management or using the hosting framework means. Communication channel establishment is initiated from the side of the calling component. Its channel management module performs queries to the knowledge-base and reasons according to the requirement of VSM

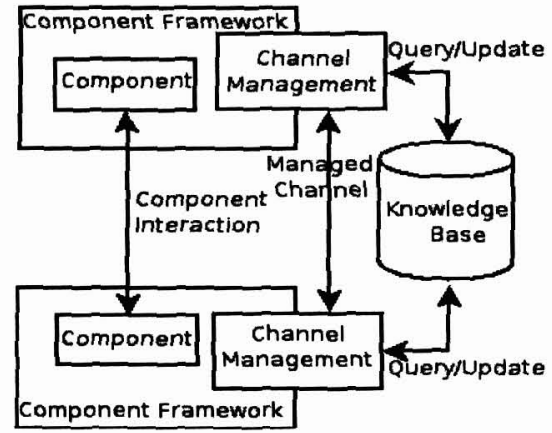

Figure 4. Component communication using shared management knowledge base

Channels have to conform the RV and RK requirements and these conditions have to be constantly maintained. For this purpose we use the abstraction of Managed Communication Channel (MCC) [8].

\subsection{Architecture}

Figure 5 shows the high-level structural representation of a Managed Communication Channel - an abstract communication medium that is able to provide transparent for the communicating components support for the VSM requirements as described in section 2.2 .

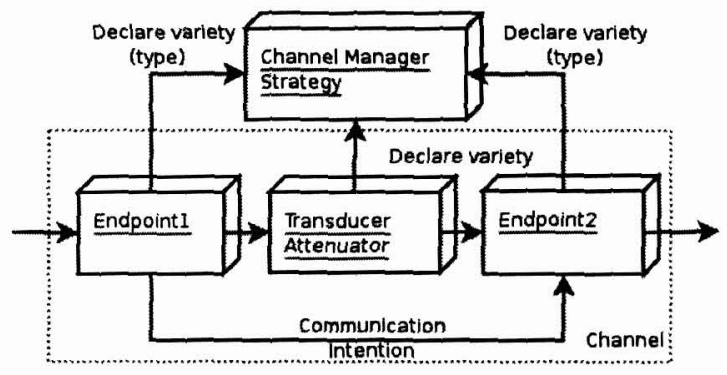

Figure. 5 Architecture of MCC

The components of MCC are:

- Endpointl - a component that intents to communicate with other components in the system.

- Endpoint2 - a component providing functional variety such as call interfaces to other components in the system

- Transducer - provides indirect communication with components.

- Channel Manager - verifies ability of the channel to transmit properly variety between Endpointl and Endpoint2. It is the component that performs $\mathrm{RV}$ verification of the transducer and the channel in general.

- Software variety - a structured data-type, consisting of identifier of the type of transmitted software element (interface, message, event, protocol, etc.) and field for specifics about the type (version or description).

The requirement for proper operation of a MCC is the endpoints and the transducer to be able to provide information about the variety they expose to the system. This variety can be any class of information specified by the used information model. In the case of component deployment these are the interfaces of services and the component version as additional variety identifier.

\subsection{Channel Composition and Activation}

MCC targets management of communication between loosely coupled and geographically distant components. This aim demands a proper sequence for binding together the channel components and their consequential activation. The general scheme of composing a MCC is shown on Figure 6. The process of channel preparation assures that all necessary components of the channel meet the needed requirements. 


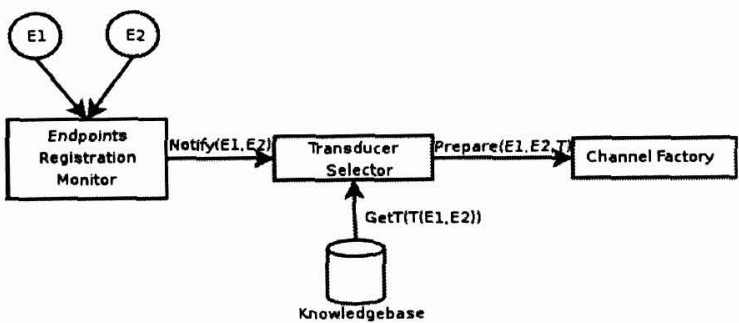

Figure 6. MCC Composition Scheme

The two endpoints are registered by a monitor which acts as a sensor for system events. The transducerselector is notified about the variety both elements expose and handle and based on this knowledge a suitable transducer is selected if needed. In case the two endpoints can communicate without mediation, a null-transducer (sends the input to the output) is selected. Selection of transducer is performed on a knowledge-base containing version compatibility and dependency records about components included in the system, as well as additional knowledge and empirical data (e.g. bugs, upgrade issues, etc.). A channel is created only if the former steps are executed successfully. After that the channel is activated and the communication between the two elements is initiated.

\section{Related Work}

There is a number of research efforts related to application of VSM in software design and project management and this approach is attracting more interest in software system engineering $[2,4,7]$. Herring [4] describes in details the architecture of VSM and its subsystem functionality regarding specification of interfaces for viable software components on the abstract level. As a pioneer in this analogy he puts strong foundation for component specification but at the same time lacks practical guidelines and it misses further development of the idea. Recent research on VSM system engineering has been done in favor of Autonomic Computing architecturing [2, 7]. The research targets system engineering using VSM and gives a good overview of the potential advantages of VSM in software system engineering. In a similar fashion we came to the idea from the roots of General System Theory and Cybernetics as instrumentation for software development and runtime phases using feedback cycles and managed communication channels [8]. This paper distinguishes itself with the further development of the concept of channel management with more practical approach to define VSM behavior in software components runtime.

\section{Conclusion and Future Work}

We have presented a model for maintenance of distributed components based and inspired by Stanford
Beer's Viable System Model. Its unique characteristics add value to existing management models with definition of component self-reference and containment, common requirements for control of variety flow, and platform independent approach for management of components on different hierarchical levels in complex nested systems. In addition to the guidelines for utilization with information models like CIM we have presented approach for channel-centric component communication to satisfy VSM requirements for viability of software systems. VSM application examples and mapping are only the beginning of a promising autonomic management technique. We have begun working on channel activation protocol specifications which together with specification of interfaces for VSM reasoning will deliver a usable platform for autonomic component communication.

\section{References}

[1] A.G. Ganek, T.A. Corbi, "The Dawning of The Autonomic Computing Era", IBM Systems Journal, Vol. 42, NO 1, 2003

[2] A. Taleb-Bendiab,D. W. Bustard, R. Sterritt, A. G. Laws, F. Keenan, Model-Based Self-Managing Systems Engineering, Proceedings of

16th International Workshop on Database and Expert Systems Applications (DEXA'05) pp. 155-159

[3] B. Melcher, B. Mitchell,Towards an Autonomic Framework: SelfConfiguring Network Services and Developing Autonomic Applications, Intel Technology Journal,Volume 08, Issue 04, November 17, 2004, ISSN 1535-864X

[4] C. Herring, S. Kaplan, The Viable System Model for Software, 4th World Multiconference on Systemics, Cybernetics and Informatics SCT 2000

[5] Carnegie Mellon Software Engineering Institute, CMMI Models and Modules, http://www.sei.cmu.edu/cmmi/models/models.html

[6] Distributed Management Task Force (DMTF), Common Management Model (CIM) Standards, http://www.dmtf.org/standards/cim

[7] D. Bustard, R. Sterritt, A. Taleb-Bendiab, A Laws, M. Randles, Frank Keenan, Towards a Systemic Approach to Autonomic Systems Engineering, Proceedings of 12th IEEE International Conference and Workshops on the Engineering of Computer-Based Systems (ECBS'05) , pp. $465-472$

[8] E. A. Stoyanov, M. A. Wischy, D. Roller, Cybernetics and General Systems Theory (GST) Principles for Autonomic Computing Design, Proceedings of 2 nd International Conference on Autonomic Computing Design ICAC05, pp. 389-390

[9] Ludwig von Bertalanffy, General System Theory, George Braziller, 1969

[10] M.Lehman, "Programs, Life Cycles and Law of Software Evolution", Proceedings of IEEE 68(9), pp. 1060 - 1076, September 1980

[11] Open Services Gateway Initiative (OSGi) Aliance, http://osgi.org

[12]Stanford Beer, Cybernetics and Management, English Universities P., 1967

[13]Stanford Beer, Diagnosing the System of Organizations, John Wiley \& Sons; 1995

[14] W. Rolls Ashby, Introduction to Cybernetics, Chapman \& Hall, London 1956 